\title{
Study and prediction of the mechanical performance of a nanotube-reinforced composite
}

\author{
F. Otero ${ }^{\mathrm{a}, \mathrm{b}, *}, \mathrm{X}$. Martínez ${ }^{\mathrm{b}, \mathrm{c}}, \mathrm{S}$. Oller ${ }^{\mathrm{a}, \mathrm{b}}$, O. Salomón ${ }^{\mathrm{b}}$ \\ ${ }^{a}$ Departamento de Resistencia de Materiales y Estructuras en la Ingeniería, ETSECCPB, Technical University of Catalonia, Spain \\ ${ }^{\mathrm{b}}$ International Center for Numerical Methods in Engineering (CIMNE), Technical University of Catalonia, Gran Capitá s/n, 08034 Barcelona, Spain \\ ${ }^{\mathrm{c}}$ Departamento de Ciencia e Ingeniería Náutica, FNB, Technical University of Catalonia, Pla de Palau 18, 08003 Barcelona, Spain
}

\section{A R T I C L E I N F O}

\section{Article history:}

Available online 10 April 2012

\section{Keywords:}

Carbon nanotubes

Composites

Mechanical properties

FEM

Constitutive model

\begin{abstract}
A B S T R A C T
Carbon nanotubes (CNTs) have been regarded as ideal reinforcements for high-performance composites. A key factor for the reinforcement efficiency is the interface bonding between the CNTs and the matrix. This paper presents a new constitutive model to predict the mechanical performance of composites made with CNTs. The model takes into account explicitly the performance of the interface between the matrix and the CNTs. The formulation developed is based in the mixing theory. It divides the composite in matrix and in a new material result of coupling the CNTs with the interface. The relation defined between interface and CNTs assumes that the load is transferred to the nanotubes along their ends and that in the central part the CNTs can develop their full strength. The composite non-linear behavior results from the non-linearities of its constituents. In case of interface damage, it also becomes non-linear the law defined to couple the interface with the CNTs. After describing the formulation, it is validated studying the elastic response of several composites made with different types of CNTs reinforcements. The non-linear behavior provided by the formulation is also studied. In both cases the numerical results are compared with experimental data showing good agreement.
\end{abstract}

Crown Copyright $\odot 2012$ Published by Elsevier Ltd. All rights reserved.

\section{Introduction}

Composites are materials made of at least two different components. Most composites consist in a matrix containing reinforcement elements [1]. The main function of matrix is to give cohesion, support the reinforcement and transfer the external actions to the reinforcements. On the other hand, the main task of the reinforcement is to improve the matrix properties.

In case of using fibers or nanotubes as reinforcement, the performance of the composite depends on the achievement of four main characteristics:

Aspect ratio of the fibers: The fiber aspect ratio is a dimensionless geometric measurement that results from dividing the length of the reinforcement by its diameter. This parameter is important because the stress distribution in the reinforcement depends on it [2]. In fibers with high aspect ratios the fiber end effect is less important. The reinforcement is considered to behave as a long fiber when its aspect ratio is larger than 1000 .

\footnotetext{
* Corresponding author at: International Center for Numerical Methods in Engineering (CIMNE), Technical University of Catalonia, Gran Capitá s/n, 08034 Barcelona, Spain.

E-mail addresses: fotero@cimne.upc.edu (F. Otero), x.martinez@upc.edu (X. Martínez), sergio.oller@upc.edu (S. Oller), salomon@cimne.upc.edu (O. Salomón).
}

Dispersion of fibers in the composite: A uniform distribution of reinforcement in the composite is fundamental to ensure that it is completely surrounded by matrix. This is necessary to obtain an effective stress transfer. A good dispersion of the reinforcement in the composite also helps to have a more uniform stress distribution in it, reducing the regions susceptible having stress concentrations.

Fiber alignment: It has been shown that the difference between random distribution and perfect alignment may represent a factor of five in the composite Young's modulus [3]. Fiber alignment also affects the isotropy of the composite, as perfect alignments increase its anisotropy.

Load transmission from the matrix to the fiber: The last and probably most important factor is the interfacial tension between matrix and reinforcement. In general, the loads in a composite structure are introduced through the matrix and are transferred to the reinforcement through the interface [2]. Therefore, the interface can be defined as the region surrounding the reinforcement where this stress transfer takes place. The properties of the composite depend on the properties of this region, and on its ability to transfer the load efficiently.

The external load applied to a composite is shared disproportionately by the different components, as their elastic properties are different. In case of considering an iso-strain hypothesis [4] 
the stresses on the reinforcement will be larger than in the matrix, as the reinforcement is stiffer than the matrix. This unequal stress distribution generates shear stresses between both materials in a region that can is usually called the interface. The load transfer from the matrix to the reinforcement is produced in this region. Shear stresses in the interface increase proportionally to the external load until a critical value, beyond which the interface breaks. This critical value is known as interfacial shear strength (IFSS) and it limits the stress transfer capacity.

Since their discovery by Lijima in 1991 [5], carbon nonotubes (CNTs) are considered a new generation of reinforcements [6]. Their "nano" size structure makes them potentially free of defects, which gives them with excellent physical properties $[7,8]$. A nanotube is a tubular cylinder formed by sp2 bonds between the carbon atoms along its length. There are two main nanotube types: single wall nanotubes (SWCNT), which are made of a single wall tube with an outer diameter in the order of $1 \mathrm{~nm}$; and multiwall nanotubes (MW/CNT), which consist in several concentric walls, one inside the other, separated by a distance of $0.34 \mathrm{~nm}$ [5]. The diameters range of MWCNT varies from 2 to $100 \mathrm{~nm}$. MWCNT can have lengths up to $100 \mu \mathrm{m}$.

Carbon nanotubes can be obtained by several procedures. The first method used was the arc-discharge [9], which consists in generating an are discharge between two graphite electrodes in an inert gas atmosphere at low pressure. The continuous electric discharge sublimates the carbon atoms of the electrodes and forms a plasma around them. This method produces free defect nano-

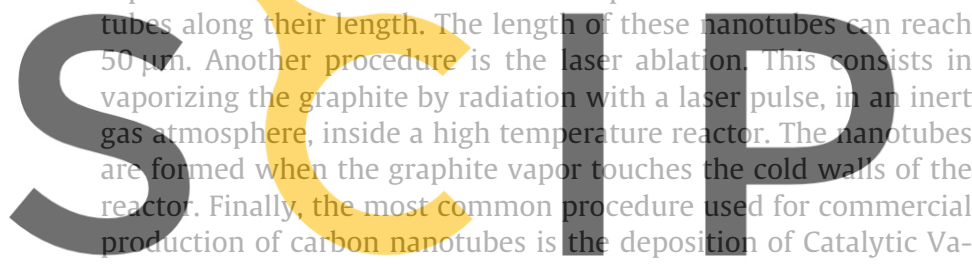
por Phase (also named, Chemical Vapor Deposition - CVD). This procedure allows producing large amounts of nanotubes at a low

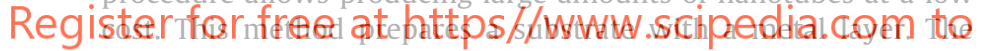

nanotube diameter depends on the size of the metal particles. The process starts by mixing two gases; one of them is used as a source of carbon, and the other for the process itself. The nanotubes grow on the side of the metal catalyst. The generated nanotubes have defects on its surface. This method can provide oriented nanotubes if there is plasma during their growth.

Nanotubes obtained by arc-discharge have Young modulus values in the order of 1 TPA. Recent measurements carried out in arc-MWCNTs (multiwall nanotubes made by arc-discharge) have provide Young modulus values with values varying from 0.27 to 0.95 TPA, ultimate strain values higher than $12 \%$, and ultimate tensile stresses in the range of 11-63 GPa [10]. In these measurements it was also obtained the stress-strain curve of the MWCNT with help an electric microscope.

The properties obtained for CVD-MWCNT (multiwall carbon nanotubes obtained by CVD) are low due to the defects in the nanotubes surface. The firsts Young modulus measurement known was made with an atomic force microscope (AFM) [11] and the values obtained were in the range of 12-50 GPa. Later on, new measurements have shown Young modulus values in order of $0.45 \mathrm{TPA}$, and ultimate tensile stresses of 3.6 GPa [12]. The lower measured values were associated with defects in the nanotube and with the slipping of the inner tubes in MWCNTs. The difference in measured values between CVD-MWCNT and arc-MWCNT shows the influence of defects on the properties of these new materials.

It is not entirely clear which nanotube type performs better as a reinforcement. A recent study made by Cadeck et al. [13] comparing the properties of a polyvinylalcohol (PVA) matrix reinforced with different types of CNTs nanotubes (double wall nanotubes
(DWCNT), SWCNT, arc-MWCNT and CVD-MWCNT) showed that the effectiveness of reinforcement is inversely proportional to its diameter, except when using SWCNT. The study also proved that the composite properties are proportional to the total interface area. The composite reinforced with SWCNT had the lowest properties; this result is associated with slipping of SWCNT inside the bundles. Finally, the study states that the best properties are obtained with the CVD-MWCNT with smaller diameter.

Currently, there are several methods that can be used to produce nanotube-reinforced composites. The choice of the most appropriate method depends of nature of the involved components [6]. All methods seek to produce a composite with a good dispersion of the CNT reinforcement and to create an interface capable of transmitting the external load to the nanotubes. The manufacturing process has to be selected taking into account that it must not affect the properties of the composite components.

Several studies have shown that the composite formation generates an interface zone around the carbon nanotubes. This interface has a different morphology and properties than the original matrix $[14,15]$. The size, shape and properties of the interface have a strong dependence on the matrix type [16] and the formation process. Pull out experimental tests indicate that IFSS values are much higher than the theoretical ones [17], which are calculated using the shear strength of the matrix. This result suggests that the interface region around the nanotube has better properties than the rest of the matrix [18]. Some studies estimate that in this region matrix properties may improve by an order of magnitude

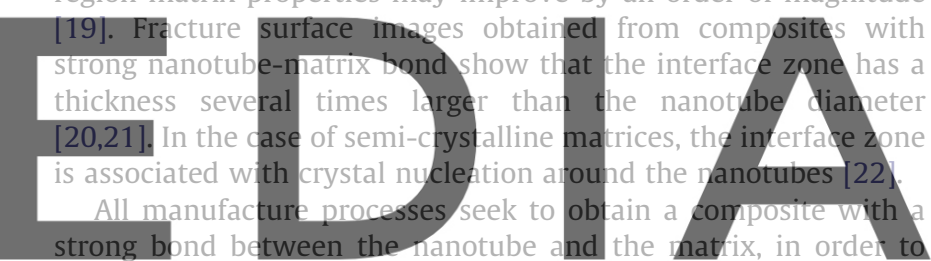
transfer effectively the loads. The IFSS defines the capacity of the bond. Values of $500 \mathrm{MPa}$ have been obtained for the IFSS when

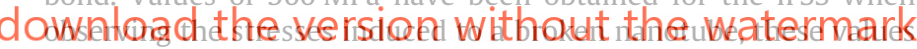
where obtained using a Transmission Electron Microscope (TEM). The study attributes this value to the presence of covalent bonds between the matrix and the nanotube [23]. Molecular Dynamics (MDs) simulations carried out confirm that strong bonds are obtained when these are covalent. In fact, the transfer load of the interface increases by an order of magnitude with just a $1 \%$ of covalent bonds in its surface [24]. On the other hand, the generation of many covalent bonds in the interface is detrimental to the intrinsic properties of the nanotube [24,25].

When there are not covalent bonds, the interaction between matrix and nanotube is made with Van der Waals forces. Several studies show that this union is weaker. Molecular Dynamics simulations made by [24] predicted values of the IFSS that do not exceed 2.8 MPa. Another study made by [26] predicted values up to $160 \mathrm{MPa}$. According to [27], the differences in the results depend on the polymer type and they can be in the range of 80-135 Mpa. The difference in the results, and the good values of IFSS, were attributed to the morphology and the capacity of the matrix to generate helical chains around the nanotube. On the other hand, nanotubes have a smoother outer surface and therefore, the contribution of the frictional forces to the IFSS are an order of magnitude lower [28].

Experimental results of pull-out tests show values of IFSS between 20 and $90 \mathrm{MPa}[19,28]$. Other experiments using the drag-out technique have shown values between 35 and $376 \mathrm{MPa}$ [18]. The disparity of the results suggests that is not always possible to generate covalent bonds. The maximum values obtained experimentally are associated to covalent bonds and consider that the interface zone has better properties than the rest of the matrix. 
Carbon nanotubes, mainly SWCNTs, tend to agglomerate. This makes very difficult to obtain a good dispersion of those in the polymer. Besides, the smooth surface of the nanotubes leads to a possible lack of bond between the nanotube and the matrix. Currently these problems are solved with a chemical functionalization of the CNTs. The covalent functionalization can be done by modifying the carboxylic acid groups on the nanotube surface and or by direct addition of reagents. The drawback of functionalizing the nanotubes is that there is an intrinsic degradation of their properties [25]. In general, two different methods have been used for the functionalization: "grafting from" and "grafting to".

The "grafting from" method is based on the initial immobilization of initiators on the nanotube surface, followed by an in situ polymerization of the suitable matrix for the formation of polymer molecules around the nanotube [29,30]. The advantage of this method is that it allows the formation of composites with a high density of nanotubes. The disadvantage is that this method requires strict control of the quantities and the conditions in which the polymerization reaction takes place.

The "grafting to" method makes the union of preformed polymer molecules to functional groups on the surface of the nanotube through chemical reactions [31,32]. The advantage of this method is that it can be used with commercial polymers. However, it has as a limitation that the initial union of the polymer chains inhibits the diffusion of macromolecules to the surface. Therefore, the density of functionalization is low.

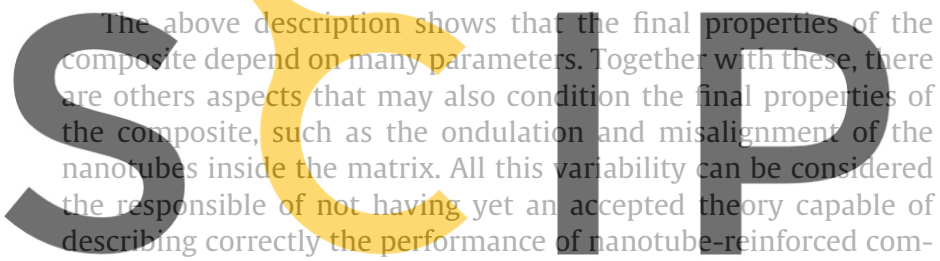
posites. It is also the reason because the existing theories fail in their predictions. Comparisons between measured mechanical

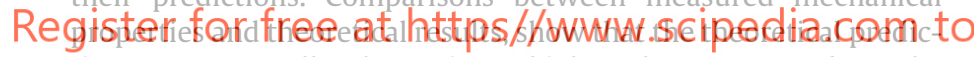
tions are generally three times higher than measured results $[21,33]$

This paper proposes a new formulation to predict the mechanical properties of nanotube-reinforced composites. The formulation is based in the mixing theory, and it obtains the properties of the composite from the mechanical performance of its constitutive materials: matrix, carbon nanotubes and the interface that bonds both of them. This new formulation is capable of predicting the response of the composite fairly accurately, requiring only the calibration of the mechanical properties of the interface.

In the following is described the constitutive model developed. Afterwards, Section 3 formulates the model and Section 4 describes its performance in the non-linear range. The implementation of the proposed formulation into a finite element code is described in Section 5. The validation of the model is shown in Section 6, where the results provided by the model are compared with experimental ones. Finally in last section some conclusions are drawn from the results obtained.

\section{Description of constitutive model}

The constitutive model presented in the following section is based in the classical mixing theory. This theory obtains the mechanical performance of the composite from the behavior of the composite constituents, each one simulated with its own constitutive law [34]. As it is written, the theory can be understood as a constitutive equation manager. The new constitutive model is formulated with the same philosophy, which increases its versatility and simulation capability.

The model assumes that the composite is the combination of three different materials: matrix, CNTs and an interface [35]. The interface corresponds to the matrix that surrounds the CNTs. It is considered as an independent component, with its own constitutive law. The interface is used to define the capacity of the matrix to transfer the loads to the reinforcement.

Although the phenomenological performance of the composite already justifies the definition of an interface material; images obtained with Scanning Electron Microscope (SEM) of CNTs reinforced composites, such the ones shown in Fig. 1, prove its actual existence. These images reveal that the structures protruding from the fractured surface have larger diameters than the original MWCNTs used in the sample preparation [20]. The material surrounding the CNTs corresponds to the interface. The presence of an interface, as a differentiable material, is also proved by Differential Scanning Calorimetry (DSC) measurements carried out in composites with a semi-crystalline polymer as matrix. These measurements show a linear increase of crystalline matrix as the nanotube volume fraction increases, suggesting that each nanotube has a crystalline coating [36].

Once having conveyed the necessity of including the interface material in a formulation to simulate the mechanical performance of CNTs reinforced composites, in the following is described the new procedure proposed, which is summarized in Fig. 2. This figure shows that the composite is divided in several layers, each one

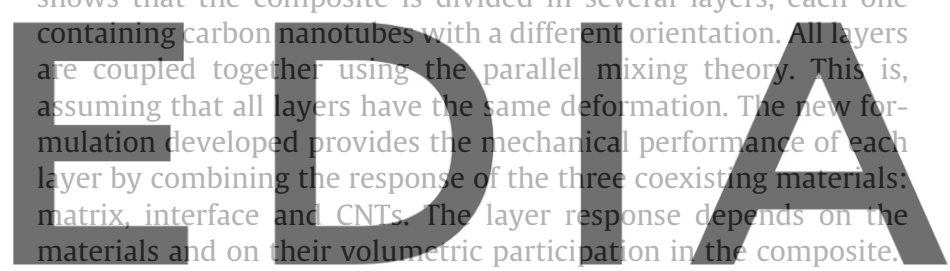

First, the composite is split into matrix and a new material that results of coupling the CNTs with the interface. The relation be-

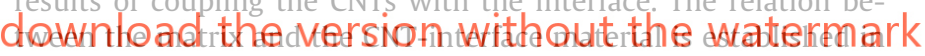

terms of the parallel mixing theory (they are assumed to have an iso-strain behavior). On the other hand, CNTs and the interface are coupled together with a combination of parallel and serial mixing theories. The serial mixing theory assumes that all components have the same stresses.

Fig. 3 shows scheme used to obtain the performance of the CNTinterface material. This is based in the short-fiber model developed by Jayatilaka [2]. According to this model, the load is transferred from the interface to the nanotube at the ends of the reinforcement, through shear stresses. In this region normal stresses in the fiber increase from zero to their maximum value, which is reached in the central part of the reinforcement. In this region there is not load transfer and shear stresses are null. This whole stress transfer scheme can be simplified assuming a CNT-interface performance defined by a serial mixing theory at the ends of the reinforcement and a parallel mixing theory at the center of it.

A parallel factor named $N^{\text {par }}$ is defined to differentiate these two regions. This parameter, multiplied by the nanotube length, provides the length of the nanotube-interface element with a parallel behavior. The length with a serial performance is defined by the complementary factor.

\section{Formulation of the constitutive model}

The Helmholtz free energy [37] of a material point subjected to small deformations can be described with the following thermodynamic formulation $[38,39]$,

$\Psi=\Psi(\varepsilon, \theta, \alpha)$ 

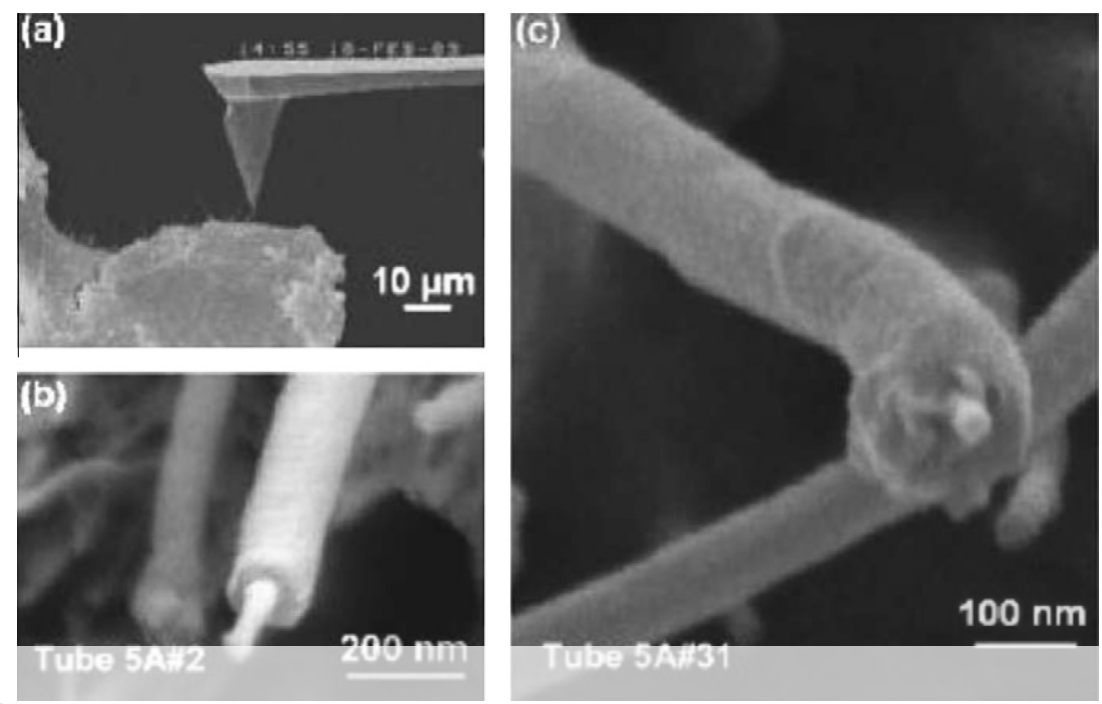

Fig. 1. SEM image of nanomanipulation and fracture surface of composites [20].

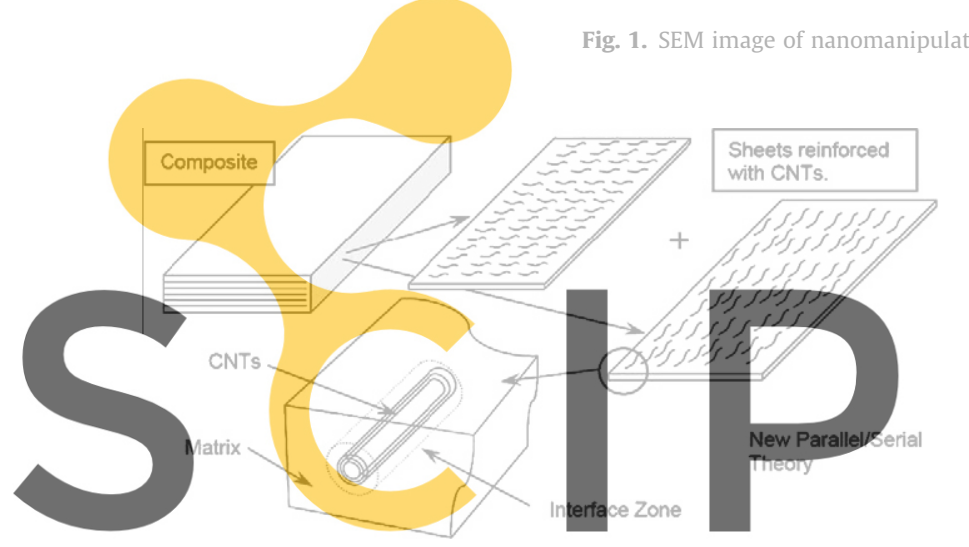

Fig. 2. Representation of formation for reinforced composite.

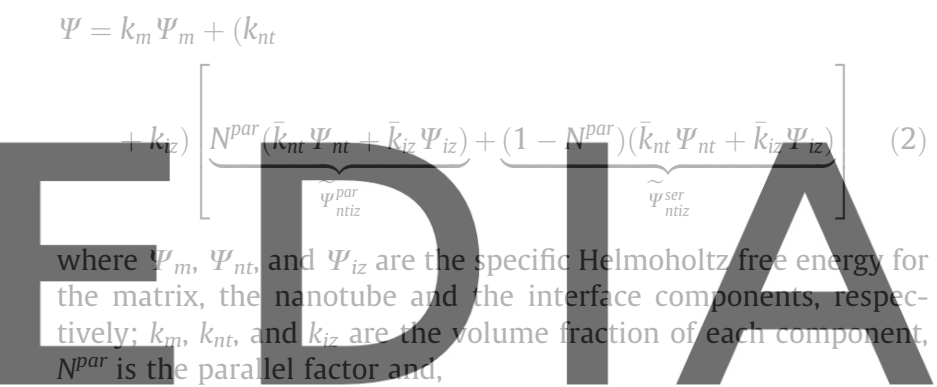

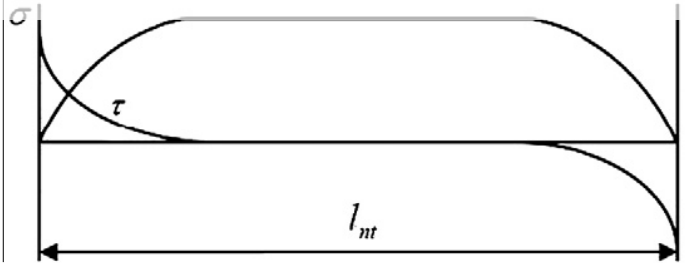

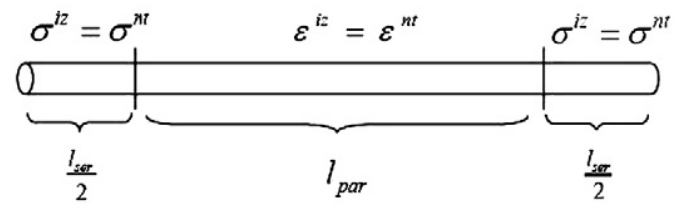

Fig. 3. Different regions in the new material CNT-interface.

where $\varepsilon$ is the deformation tensor, $\theta$ a measure of temperature and $\alpha=\left\{\varepsilon^{p}, d, s\right\}$ a set of inner variables, for example: $\varepsilon^{p}$ is the plastic deformation, $d$ damage inner variable and $s$ any other material internal variables.

The model proposed to simulate the composite combines the different components using the serial and parallel mixing theories. If this combination is performed according to what has been described in previous section, the expression of the Helmholtz free energy may be written as:

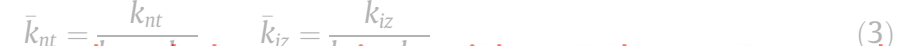
own load the versiokiz without the watermark are the volume fractions of the carbon nanotubes and the interface in the new CNT-interface material. These volume fractions must verify:

$k_{m}+k_{n t}+k_{i z}=1 \quad \bar{k}_{n t}+\bar{k}_{i z}=1$

The relation among the strain tensors of the different components is:

$\varepsilon=\varepsilon_{m}=\varepsilon_{n t i z}^{p a r}=\varepsilon_{n t i z}^{\text {ser }}$

being $\varepsilon$ and $\varepsilon_{m}$ the composite and matrix deformations, respectively; $\varepsilon_{n t i z}^{p a r}$ the deformation the new CNT-interface material with a parallel behavior; and $\varepsilon_{n t i z}^{\text {ser }}$ the deformation of the CNT-interface material with a serial behavior.

The tangent constitutive tensor of the composite material may be derived from Eq. (2),

$C=\frac{\partial^{2} \Psi}{\partial \varepsilon \otimes \partial \varepsilon}=k_{m} \frac{\partial^{2} \Psi_{m}}{\partial \varepsilon_{m} \otimes \partial \varepsilon_{m}}+\frac{\partial^{2} \widetilde{\Psi}_{n t i z}^{p a r}}{\partial \varepsilon_{n t i z}^{p a r} \otimes \partial \varepsilon_{n t i z}^{p a r}}+\frac{\partial^{2} \widetilde{\Psi}_{n t i z}^{s e r}}{\partial \varepsilon_{n t i z}^{s e r} \otimes \partial \varepsilon_{n t i z}^{s e r}}$

A parallel behavior means that all composite constituents have the same strain value. Therefore:

$$
\begin{aligned}
\varepsilon_{n t i z}^{\text {par }} & =\varepsilon_{n t}=\varepsilon_{i z} \Rightarrow \frac{\partial^{2} \widetilde{\Psi}_{n t i z}^{\text {par }}}{\partial \varepsilon_{n t i z}^{\text {par }} \otimes \partial \varepsilon_{n t i z}^{p a r}}=N^{\text {par }}\left[\bar{k}_{n t} C_{n t}+\bar{k}_{i z} C_{i z}\right] \\
& =N^{\text {par }} C_{n t i z}^{\text {par }}
\end{aligned}
$$

and a serial behavior means that all composite constituents have the same stress value. Thus: 
$\sigma_{n t i z}^{\text {ser }}=\sigma_{n t}=\sigma_{i z} \Rightarrow \varepsilon_{n t}=C_{n t}^{-1}: C_{n t i z}^{\text {ser }}: \varepsilon_{n t i z}^{\text {ser }} ; \quad \varepsilon_{i z}=C_{i z}^{-1}: C_{n t i z}^{\text {ser }}: \varepsilon_{n t i z}^{\text {ser }}$

$\frac{\partial^{2} \widetilde{\Psi}_{n t i z}^{\text {ser }}}{\partial \varepsilon_{n t i z}^{\text {ser }} \otimes \partial \bar{\varepsilon}_{n t i z}^{\text {ser }}}=\left(1-N^{p a r}\right)\left[\bar{k}_{n t} C_{n t}^{-1}+\bar{k}_{i z} C_{i z}^{-1}\right]^{-1}=\left(1-N^{p a r}\right) C_{n t i z}^{\text {ser }}$

Replacing Eqs. (7) and (9) in Eq. (6) it is possible to obtain a simplified expression of the tangent constitutive tensor:

$C=k_{m} C_{m}+\left(k_{n t}+k_{i z}\right)\left[N^{p a r} C_{n t i z}^{p a r}+\left(1-N^{p a r}\right) C_{n t i z}^{\text {ser }}\right]$

\subsection{Definition of the parallel factor}

The parallel factor is defined as,

$N^{\text {par }}=\frac{l_{\text {par }}}{l_{n t}}, \quad 0 \leqslant N^{\text {par }} \leqslant 1$

where $l_{n t}$ is the length of the nanotube and $l_{p a r}$ is function of geometry and mechanical properties of the nanotube and the interface. The value of this length can be obtained from the equation of tension distribution in a reinforcement considering perfect bond with the matrix, which is [2]
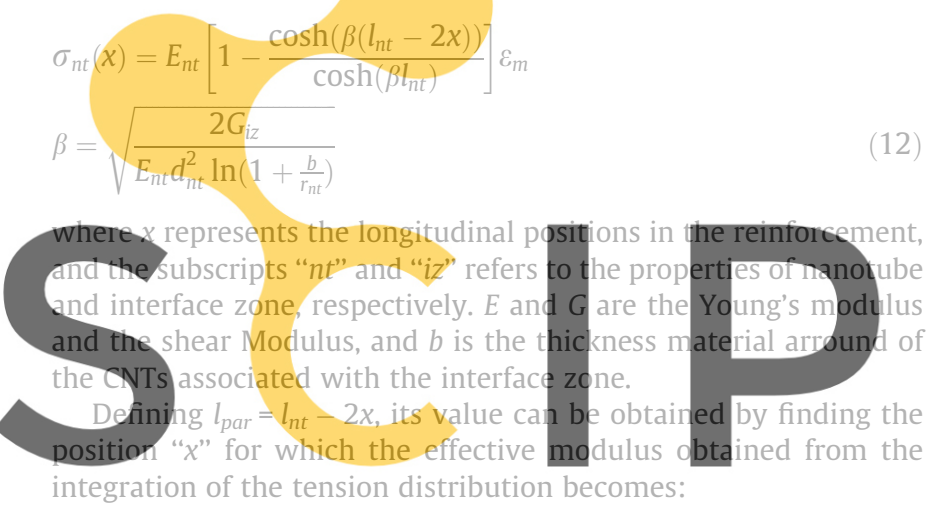

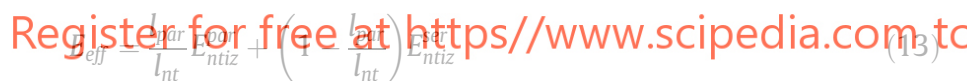

This procedure provides a value of the parallel length of:

$$
l_{p a r}=\frac{1}{\beta} \cosh ^{-1}\left[\frac{1}{3} \cosh \left(\beta l_{n t}\right)\right]
$$

\subsection{Definition of the volume fraction of the interface region}

Based on the results reported in [36], the interface zone can be considered the region surrounding the carbon nanotube in which an amorphous matrix becomes crystalline. The volume fraction of the interface zone can be obtained as:

$\chi_{c}=\chi_{o}+k_{i z}$

where $\chi_{c}, \chi_{o}$ are the volume fractions of crystalline matrix with and without CNTs, respectively. Assuming that the interface zone is a cylinder around the CNTs, it is possible to relate the volume fraction of the interface zone with the parameter $\frac{b}{r_{n t}}$.

$$
\begin{aligned}
k_{i z} & =\frac{N\left(\pi r^{2} l_{n t}-\pi r_{n t}^{2} l_{n t}\right)}{V}=\frac{N \pi r_{n t}^{2} l_{n t}}{V}\left[\left(\frac{r}{r_{n t}}\right)^{2}-1\right] \\
& =k_{n t}\left[\left(\frac{r}{r_{n t}}\right)^{2}-1\right]
\end{aligned}
$$

where $V$ is the total composite volume, $r$ is the radius of interface zone and $N$ is the total number of nanotubes in the composite.

The relation between the radius of the nanotube and the interface is obtained replacing Eq. (16) in Eq. (15): $\frac{r}{r_{n t}}=+\sqrt{\frac{\left(\chi_{c}-\chi_{0}\right)}{k_{n t}}+1}, \quad \chi_{c} \geqslant \chi_{0}$

and;

$\frac{r}{r_{n t}}=1+\frac{b}{r_{n t}} \Rightarrow \frac{b}{r_{n t}}=+\sqrt{\frac{k_{i z}}{k_{n t}}+1}-1, \quad k_{\text {iz }} \geqslant 0$

\subsection{Constitutive model for a single material}

The formulation developed require all composite components to fulfill Eq. (1). Therefore, it is possible to use any constitutive law to describe the mechanical performance of the different components. However, for the sake of simplicity, in the following are defined the three specific models that will be used for each composite component.

\subsubsection{Constitutive model for matrix material}

Matrix material is defined with an elastoplastic law. The specific Helmholtz free energy for this material, considering uncoupled elasticity is:

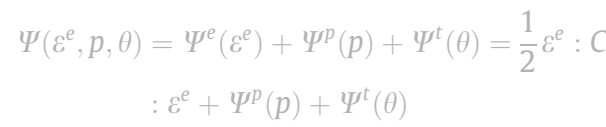

where $\Psi^{e}$ is the specific elastic free energy, $\Psi^{p}$ is the specific plastic

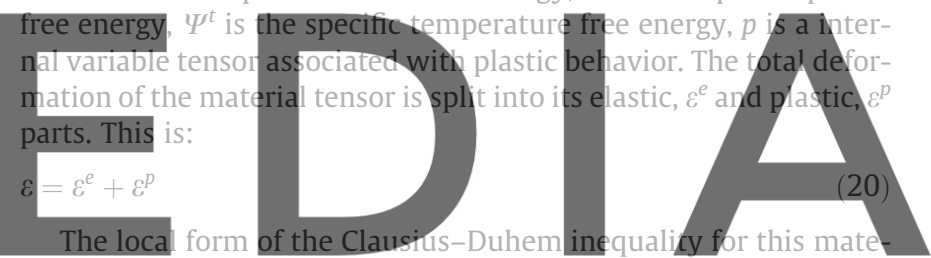

rial can be expressed as:

\section{clownload the yersgiera without the watermark \\ $\sigma:\left(\dot{\varepsilon}^{e}+\dot{\varepsilon}^{p}\right)-\eta \dot{\theta}-\left[\frac{\partial \Psi^{e}}{\partial \varepsilon^{e}}: \dot{\varepsilon}^{e}+\frac{\partial \Psi^{p}}{\partial p} \cdot \dot{p}+\frac{\partial \Psi^{t}}{\partial \theta} \dot{\theta}\right]-\frac{1}{\theta} q \cdot \frac{\partial \theta}{\partial x} \geqslant 0 \quad$ (22)$$
\left(\sigma-\frac{\partial \Psi^{e}}{\partial \varepsilon^{e}}\right): \dot{\varepsilon}^{e}-\left(\eta+\frac{\partial \Psi^{t}}{\partial \theta}\right) \dot{\theta}+\sigma: \dot{\varepsilon}^{p}-\frac{\partial \Psi^{p}}{\partial p} \cdot \dot{p}-\frac{1}{\theta} q \cdot \frac{\partial \theta}{\partial x} \geqslant 0
$$

being $\sigma$ the stress tensor, $\eta$ the entropy, and $q$ the vector field of heat flow. To ensure compliance with the second thermodynamic law it must be defined,

$\sigma \doteq \frac{\partial \Psi^{e}}{\partial \varepsilon^{e}} \quad \eta \doteq-\frac{\partial \Psi^{t}}{\partial \theta} \quad P \doteq-\frac{\partial \Psi^{p}}{\partial p}$

where $P$ is the thermodynamic tensor associated with the internal variable tensor $p$. Finally, the mechanical dissipation for a material point is,

$\Xi_{m}=\Xi_{p}=\sigma: \dot{\varepsilon}^{p}+P \cdot \dot{p} \geqslant 0$

\subsubsection{Constitutive model for interface material}

The interface region is simulated with a damage material. In this case, the expression of the Helmholtz free energy is,

$$
\begin{aligned}
\Psi(\varepsilon, d, \theta) & =\Psi^{e}(\varepsilon, d)+\Psi^{t}(\theta)=(1-d) \Psi_{o}^{e}(\varepsilon)+\Psi^{t}(\theta) \\
& =(1-d) \frac{1}{2} \varepsilon: C: \varepsilon+\Psi^{t}(\theta)
\end{aligned}
$$

where $d$ is a internal variable associated with the damage. The local form of the Clausius-Duhem inequality Eq. (21) for this material can be expressed as, 
$\sigma: \dot{\varepsilon}-\eta \dot{\theta}-\left[\frac{\partial \Psi^{e}}{\partial \varepsilon}: \dot{\varepsilon}+\frac{\partial \Psi^{e}}{\partial d} \dot{d}+\frac{\partial \Psi^{t}}{\partial \theta} \dot{\theta}\right]-\frac{1}{\theta} q \cdot \frac{\partial \theta}{\partial x} \geqslant 0$

$\left(\sigma-\frac{\partial \Psi^{e}}{\partial \varepsilon}\right): \dot{\varepsilon}-\left(\eta+\frac{\partial \Psi^{t}}{\partial \theta}\right) \dot{\theta}-\frac{\partial \Psi^{e}}{\partial d} \dot{d}-\frac{1}{\theta} q \cdot \frac{\partial \theta}{\partial x} \geqslant 0$

To ensure compliance with the second thermodynamic law it must be defined:

$\sigma \doteq \frac{\partial \Psi^{e}}{\partial \varepsilon} \quad \eta \doteq-\frac{\partial \Psi^{t}}{\partial \theta} \quad D \doteq-\frac{\partial \Psi^{e}}{\partial d}$

being $D$ the thermodynamic scalar associated with the internal scalar variable $d$. And, the mechanical dissipation for a material point is:

$\Xi_{m}=\Xi_{d}=D \cdot \dot{d} \geqslant 0$

\subsubsection{Constitutive model for nanotubes}

Nanotubes are considered elastic. In this case the Helmholtz free energy can be written as,

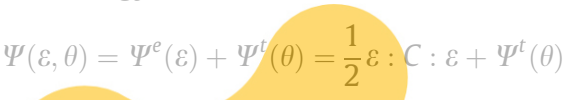

and the local form of the Clausius-Duhem inequality Eq. (21) can be expressed in this case as,
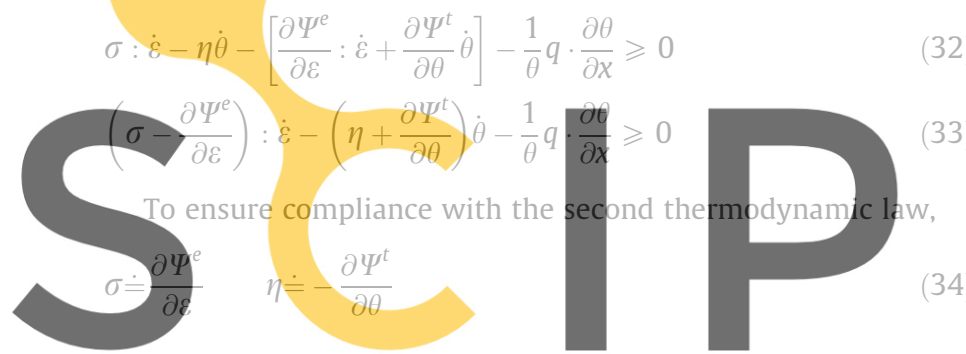

3.3.4. Equivalent properties for MWCNTS

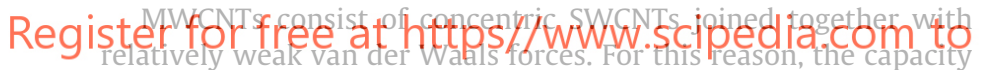

to transfer the load from the external wall to the internal walls is low. Some papers $[40,41]$ propose to simulate the CNTs like a solid cylinder with same exterior diameter and length, but with effective properties. The effective properties are obtained assuming that the outer wall takes the total load. In this approach it is assumed that the properties of the outer wall correspond to those of a graphite sheet. The effective stiffness of the MWCNT is calculated by imposing that for a same applied force, the deformation must be the same:

$\bar{\varepsilon}_{n t}=\varepsilon_{n t} \Rightarrow \bar{E}_{n t}=\frac{A_{o w}}{\bar{A}_{n t}} E_{g}$

where $\bar{E}_{n t}$ and $E_{g}$ are the Young's modulus of the effective solid nanotube and graphite sheet, respectively, and $\bar{A}_{n t}$ and $A_{o w}$ are the areas of the effective solid nanotube and outer wall, respectively. Eq. (35) can be also read as,

$\bar{E}_{n t}=\left[1-\left(1-\frac{2 t}{d_{n t}}\right)^{2}\right] E_{g}, \quad \frac{t}{d_{n t}} \leqslant 0.5$

being $t$ the thickness of one wall in the MWCNT and $d_{n t}$ is the external diameter of the MWCNT.

Using the same procedure it is possible to obtain the shear modulus of the solid cylinder, by forcing the same twist when applying the same torque $(T)$.

$\bar{\phi}_{n t}=\phi_{n t} \Rightarrow \frac{T l_{n t}}{\bar{G}_{n t} \bar{J}_{n t}}=\frac{T l_{n t}}{G_{g} J_{o w}} \Rightarrow \bar{G}_{n t}=\frac{J_{o w}}{\bar{J}_{n t}} G_{g}$ where $\bar{G}_{n t}$ and $G_{g}$ are the shear modulus of the effective solid CNTs and graphite sheet, respectively, and $\bar{J}_{n t}$ and $J_{o w}$ are the polar moment of inertia of the effective solid CNTs and outer wall, respectively.

$\bar{J}_{n t}=\frac{\pi d_{n t}^{4}}{32}, \quad J_{o w}=\frac{\pi\left(d_{n t}^{4}-\left(d_{n t}-2 t\right)^{4}\right)}{32}$

Replacing the expressions of Eq. (38) in Eq. (37), the equivalent shear modulus can be written as,

$\bar{G}_{n t}=\left[1-\left(1-\frac{2 t}{d_{n t}}\right)^{4}\right] G_{g}$

Finally, it is necessary to obtain the new density of the effective solid CNTs, as the total weight of the MWCNTs cannot change in the composite when they are considered a solid cylinder.

$\bar{\rho}_{n t}=\frac{A_{n t}}{\bar{A}_{n t}} \rho_{g} \Rightarrow \bar{\rho}_{n t}=\left[1-\left(\frac{d_{i}}{d_{n t}}\right)^{2}\right] \rho_{g}$

being $\rho_{g}$ the density of the graphite sheet $\left(\rho_{g}=2.25\left[\mathrm{~g} \mathrm{~cm}^{-3}\right]\right)$ and $d_{i}$ the internal diameter of the MWCNTs.

The most common parameter used to define the amount of CNTs added to a composite is their weight fraction. However, the numerical model developed requires knowing the volume fraction. The volumen fraction of CNTs in the composite is the volume that occupies a solid cilinder with the same external

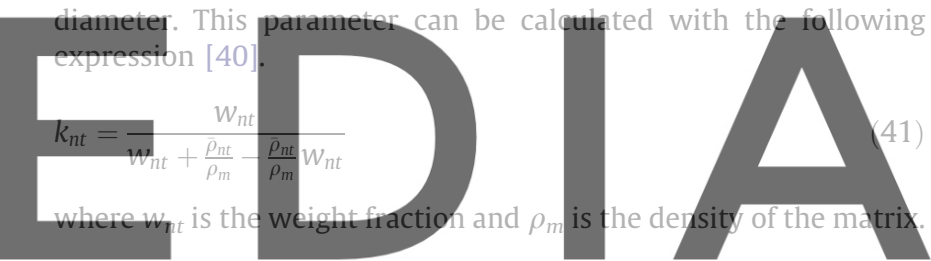

4. Material non-linearity of the proposed model

\section{dowpload the version without the watermark}

from the mechanical response of its constituent materials, and each constituent is simulated with its own constitutive law. Therefore, if a constituent (i.e. the interface) is simulated with a non-linear law, the whole composite will become non-linear. As it has been already explained, with the present model it is possible to use any non-linear formulation to simulate the constituents, such as plasticity, damage, and viscosity.

Besides the non-linear performance provided by each constituent, the load transfer capacity of the interface region is also affected if the interface is damaged. This effect must be included in the formulation.

According to Fig. 3, the load is transferred from the interface to the CNTs reinforcements at their ends. Interface damage is expected to occur at the ends of the reinforcement, where there is larger stress concentrations. Assuming that the damaged region is unable to transfer loads and that the length required to transfer loads must remain constant, interface damage ends up affecting the parallel length of the nanotube, which can be calculated as:

$l_{\text {par }}=l_{\text {par }}^{o}(1-d)$

where $l_{\text {par }}^{0}$ is the initial length of the nanotube working in parallel and $d$ is the interface damage.

The dependence of the parallel length on the interface material damage provides a non-linear response of the composite, even when matrix and the carbon nanotube reinforcement are in their linear range. 


\section{Numerical implementation}

The proposed model has been implemented in PLCd [42], a finite element code that works with $3 \mathrm{D}$ solid geometries. The algorithm developed is described in Fig. 4. PLCd has already implemented the constitutive laws that will be used to predict the performance of the composite components (elasto-plastic, elastodamage, and elastic). The formulation proposed has been written so that the constitutive laws of the constituents are seen as "black boxes", following the recommendations of $[43,44]$.

The FEM code enters into the new formulation with the prediction of the strain of the composite material in the actual time step. Layers are assumed to have all the same strain; therefore the strain tensor of each layer is obtained rotating the composite strain to the direction in which the CNTs are oriented. In each layer, the strain of the matrix and the CNTs-interface are the same, as they work in parallel (Eq. (5)). Knowing the strains for matrix material it is possible to obtain its stresses straightforward. On the other hand, to obtain the stresses for the CNTinterface material, it is necessary to separate it in two regions. In the flow chart (Fig. 4), these two regions are represented as "Parallet Block" and "Serial Block". This division is performed based on the value of $N^{\text {par }}$ (defined in Eq. (11)). This value depends on the damage evolution of the interface, as has been explained in Section 4.

The Parallel Block corresponds to the central region, where the CNTs and the interface work in parallel and, therefore, they have

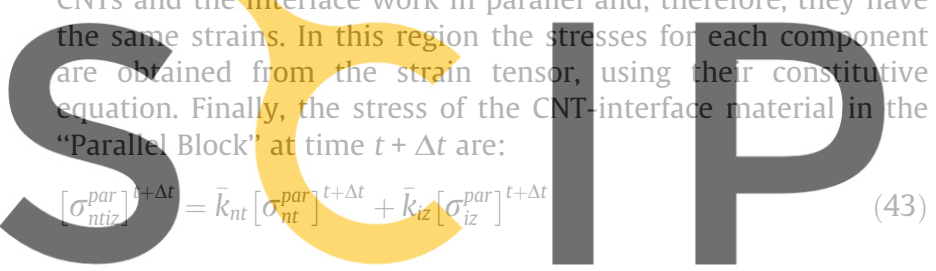

On the other hand, at the ends of the CNTs, the interface-CNTs material has a serial behavior and it is necessary an initial prediction of the CNT or of the interface strains, in order to integrate the local stress in both components. If this initial prediction is made on the interface, its strains can be computed as:

$\Delta \varepsilon_{n t i z}^{\text {ser }}=\left[\varepsilon_{n t i z}^{\text {ser }}\right]^{t+\Delta t}-\left[\varepsilon_{\text {ntiz }}^{\text {ser }}\right]^{t}$

$\left[\Delta \varepsilon_{i z}^{\text {ser }}\right]_{o}=\left[C_{i z}^{\text {ser }}\right]^{-1}: C_{n t i z}^{\text {ser }}: \Delta \varepsilon_{n t i z}^{\text {ser }}$

$\left[\varepsilon_{i z}^{\text {ser }}\right]_{o}^{t+\Delta t}=\left[\varepsilon_{i z}^{\text {ser }}\right]^{t}+\left[\Delta \varepsilon_{i z}^{\text {ser }}\right]_{0}$

and the strain of the interface in the iteration step $n$ is used to calculate the strain of the CNT:

$\varepsilon_{n t i z}^{s e r}=\bar{k}_{n t} \varepsilon_{n t}^{s e r}+\bar{k}_{i z} \varepsilon_{i z}^{s e r}$

$\left[\varepsilon_{n t}^{\text {ser }}\right]_{n}=\frac{1}{\bar{k}_{n t}}\left[\varepsilon_{n t i z}^{\text {ser }}\right]-\frac{\bar{k}_{i z}}{\bar{k}_{n t}}\left[\varepsilon_{i z}^{\text {ser }}\right]_{n}$

Once knowing the strains of both component materials, the constitutive law of each one is used to calculate their stress tensor: Afterwards it is necessary to verify that the iso-stress condition is indeed fulfilled.

$\left[\Delta \sigma^{\text {ser }}\right]_{n}=\left[\sigma_{i z}^{\text {ser }}\right]_{n}-\left[\sigma_{n t}^{\text {ser }}\right]_{n} \leqslant$ tolerance

If the residual stress is greater than the tolerance, the prediction of the interface deformation must be corrected. A Newton-Raphson scheme is adopted to do this correction. The method uses the Jacobian to update the unknown variable, in this case, the interface strain.
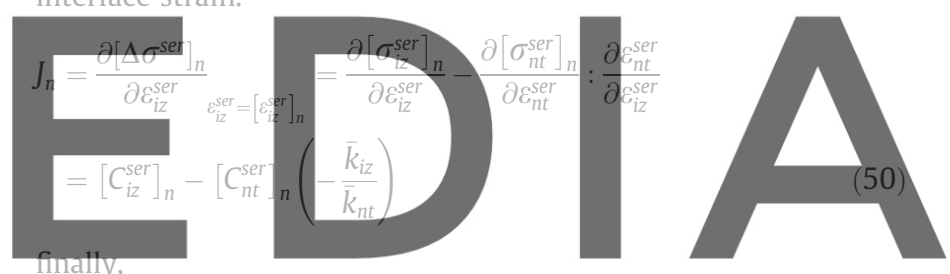

Register for free a ${ }_{\varepsilon_{c}}$ tht

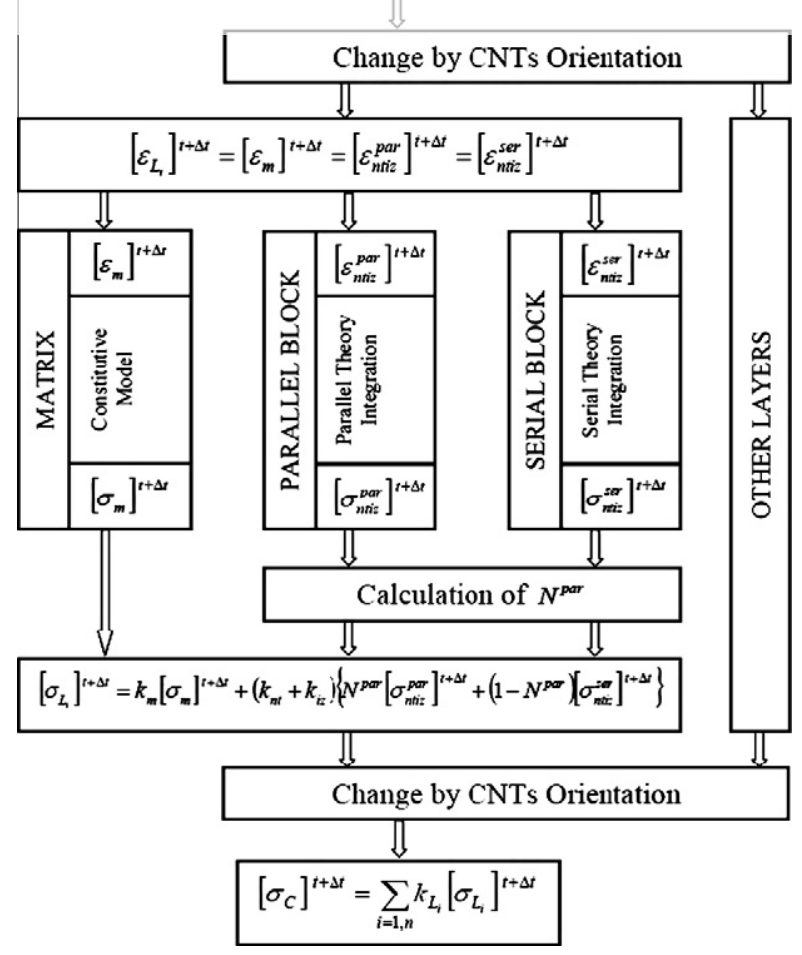

Fig. 4. Flow chart of the proposed model in a FEM code. and the strain of the interface for the next step $n+1$ is estimated as,

$\left[\varepsilon_{i z}^{s e r}\right]_{n+1}=\left[\varepsilon_{i z}^{s e r}\right]_{n}-J_{n}^{-1}:\left[\Delta \sigma^{s e r}\right]_{n}$

This iterative process continues until the residual stress is smaller than the required tolerance. The final stresses in the serial region (Serial Block) of the CNTs-interface are:

$\left[\sigma_{n t i z}^{\text {ser }}\right]^{t+\Delta t}=\left[\sigma_{n t}^{\text {ser }}\right]^{t+\Delta t}=\left[\sigma_{i z}^{\text {ser }}\right]^{t+\Delta t}$

and the final stress tensor for a specific layer is obtained as:

$$
\begin{aligned}
{[\sigma]^{t+\Delta t}=} & k_{m}\left[\sigma_{m}\right]^{t+\Delta t}+\left(k_{n t}\right. \\
& \left.+k_{i z}\right)\left\{\left[N^{p a r}\right]^{t+\Delta t}\left[\sigma_{n t i z}^{p a r}\right]^{t+\Delta t}+\left[1-N^{p a r}\right]^{t+\Delta t}\left[\sigma_{n t i z}^{\text {ser }}\right]^{t+\Delta t}\right\}
\end{aligned}
$$

\section{Results}

\subsection{Validation of the elastic response provided by the formulation}

In the following are compared the composite stiffness predicted by the proposed formulation with experimental values obtained from the literature. Coleman et al. $[35,45]$ tested several composites made of the same matrix with different MWCNTs. The matrix material was polyvinyl alcohol (PVA) and its Young's modulus was $E_{m}=1.9 \pm 0.3$ (GPa).

The authors found that the Young's modulus of the crystalline polymer phase was $E_{i z}=46$ (GPa) and the parameter $\frac{b}{r_{n t}}$ was estimated following the same procedure described in Section 3.2. 
The nanotubes used in [35] were an arc grown MWCNT (ArcMWCNT), two types of catalytic MWCNT from Nanocyl S.A. (CVD-1, CVD-2), a catalytic MWCNT produced in Orléans (France) (CVD-3), and a double walled nanotube (Dwnt). In [45] the nanotubes used were MWCNT from Nanocyl S.A. (MWCNT).

The maximum Young's modulus of the CNTs is $\sim 1$ (TPa) [35], which corresponds to the stiffness of a perfect graphite sheet. This is the value used in the model validation. The equivalent stiffness of the nanotube is calculated considering a thickness of the outer layer of $t=0.34(\mathrm{~nm})[5,40]$.

The most important data of the nanotubes used is presented in Table 1:

A parameter missing in Table 1 is the direction distributions of the CNT. In general, obtaining this information is very complicated. To outstep this impediment it is possible to rewrite Eq. (10) for one layer as,

$C_{\text {layer }}=k_{m} C_{m}+k_{n t i z} C_{n t i z}^{\text {eff }}$

where

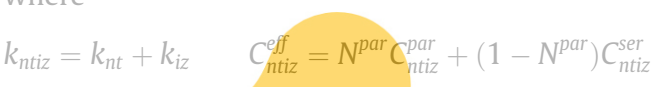

Cox and Krenchel modified the rule of mixtures proposing the following equation to calculate the composite Young's modulus,

$E=k_{m} E_{m}+k_{f} \eta_{0} E_{e f f}$

where $E_{m}$ and $E_{\text {eff, }}$ are the Young's modulus of the matrix and effective reinforcement, respectively. The volume fraction for each com-
ponert is $k$ and $\eta_{0}$ is a fiber orientation efficiency factor.
For the present validation Eq. (57) will be modified, adapting it
to the developed formulation. Therefore,
$C_{\text {conposite }}=k_{m} C_{m}+k_{n t i z} \eta_{0} C_{n t i z}$
The value of the efficiency factor related to fiber orientation was taken from literature. In composites with a randon $\eta_{0}=0.38$

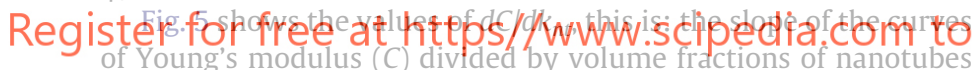
$\left(k_{n t}\right)$, for the different composites considered. In the figure the short lines represent the limits of the range experimental results presented in $[35,45]$ and the red ${ }^{1}$ points correspond to the numerical result for each CNT type, obtained with the formulation proposed in this paper.

This figure shows that the formulation is capable of predicting the elastic stiffness of the composite, as most of the values obtained are comprehended between the limits defined by the experimental tests. There is only one case in which the value obtained exceed the limits of the numerical test. This is because the effective Young's modulus of the Dwnt is highest since its diameter is really low.

\subsection{Validation of the non-linear performance of the formulation}

The non-linear behavior of the numerical model has been validated comparing the results provided by the model with the experimental data obtained from the paper of Meng et al. [46]. In this paper the matrix used was Polyamide 6 (PA6) and all composites contained a $1 \mathrm{wt} . \%$ of MWCNTs reinforcement.

The MWCNTs used in the experimental tests were purchased from Chengdu Organic Chemistry Co. Ltd. Two different composites where manufactured with these nanotubes. One of them contains the nanotubes "as is", without any previous treatments. These nanotubes are called U-MWCNT. The other composite uses nano-

\footnotetext{
${ }^{1}$ For interpretation of color in Figs. 5-8, the reader is referred to the web version of this article.
}

Table 1

Relevant data of the nanotubes used by Coleman et al. [35,45].

\begin{tabular}{lllrrrl}
\hline Type & $d_{n t}(\mathrm{~nm})$ & $l_{n t}(\mu \mathrm{m})$ & $l_{n t} / d_{n t}$ & $b / r_{n t}$ & $\bar{E}_{n t}(\mathrm{GPa})$ & $N^{\text {par }}$ \\
\hline Arc-MWCNT & 24 & 1 & 42 & 0.81 & 56 & 0.97 \\
CVD-3 & 16 & 3.8 & 238 & 1.47 & 83 & 0.99 \\
CVD-2 & 14 & 2.1 & 150 & 2.27 & 95 & 0.99 \\
CVD-1 & 15 & 1.8 & 120 & 2.83 & 89 & 0.98 \\
Dwnt & 2.5 & 2.2 & 880 & 4.87 & 470 & 0.99 \\
MWCNT & 15 & 1.72 & 115 & 3.30 & 89 & 0.98 \\
\hline
\end{tabular}

tubes that where treated with a mixture of concentrated sulfuric and nitric acids. These are called A-MWCNT.

Numerical simulations of molecular structural mechanics of CNTs show that the Young's moduli are in the range of $1.05 \pm 0.05$ (TPa) and the shear moduli is about $0.4 \pm 0.05$ (TPa) [47]. It has been also shown that these values do not change significantly for CNTs with two, three or four walls.

Regarding the transverse modulus of CNTs, it has been assessed from numerical and experimental results that there is an inverse relationship between axial and transverse modulus for carbon fibers [48]. Higher axial stiffness is associated to a longer and more aligned crystalline structure of the nanotube in this direction, which reduces properties in the transverse direction. Following this approach, in current simulation the transverse moduli of the MWCNTs is defined with the same value as the interface.

Matrix material is characterized with an isotropic, elasto-plastic model using a Von-Mises yield criterion. The mechanical parame-

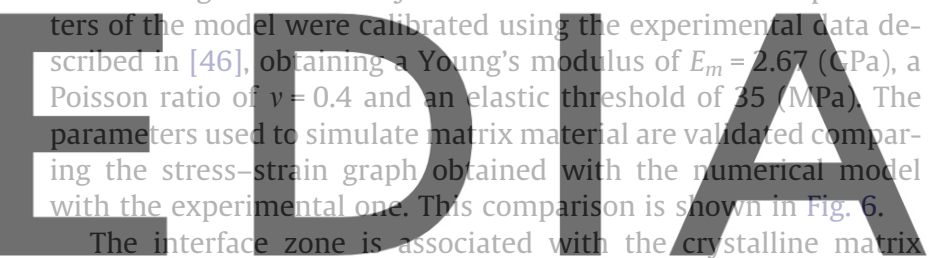
around of MWCNTs. The properties of this material are better than those of the amorphous matrix. The volume fraction of the inter-

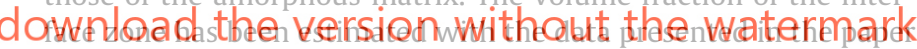
of Meng [46] and the equations developed in Section 3.2. On the other hand, the mechanical properties of the interface are used to calibrate the model. In current simulation, the interface has been defined with a isotropic, elasto-damage model with linear softening and a Tresca yield surface. The mechanical parameters used are $E_{i z}=5(\mathrm{GPa})$ and $v_{i z}=0.4$. Damage in the interface starts for a stress threshold of 120 (MPa). This value is in the range of theoretical and experimental tests value obtained in [17].

Finally, the equivalent properties of the MWCNTs were obtained using the equations described in Section 3.3.4. The diameter of MWCNT is $d_{n t}=50(\mathrm{~nm})$. The measurement of several MWCNTs provided an estimation of the internal diameter of $d_{i}=8.2(\mathrm{~nm})$ [40]. The effective density of MWCNTs has a value of $\bar{\rho}_{n t}=2.2\left(\mathrm{~g} \mathrm{~cm}^{-3}\right)$; and the volume fraction of MWCNTs in the composite is $0.51 \%$. The MWCNTs have been simulated using an anisotropic elastic material with the following properties:

$E_{1 n t}=\bar{E}_{n t}=56(\mathrm{GPa}), E_{2 n t}=E_{3 n t}=E_{i z}=5(\mathrm{GPa})$

$G_{12 n t}=G_{13 n t}=\bar{G}_{n t}=41(\mathrm{GPa}), G_{23}=1.8(\mathrm{GPa})$

$v_{12 n t}=v_{13 n t}=v_{23 n t}=v_{n t}=0.2$

$v_{i j}=\frac{E_{i}}{E_{j}} v_{j i} \Rightarrow v_{21 n t}=v_{31 n t}=0.018 \quad v_{32 n t}=0.2$

The composites tested had a random distribution of the MWCNTs. This is simulated in the numerical model by dividing the composite in several layers, each one containing CNTs with a different orientation. Current simulation divides the composite in 10 layers and CNTs angles varying from $0^{\circ}$ to $90^{\circ}$. Each layer has a volume fraction of $10 \%$. Table 2 shows the volume fractions of 


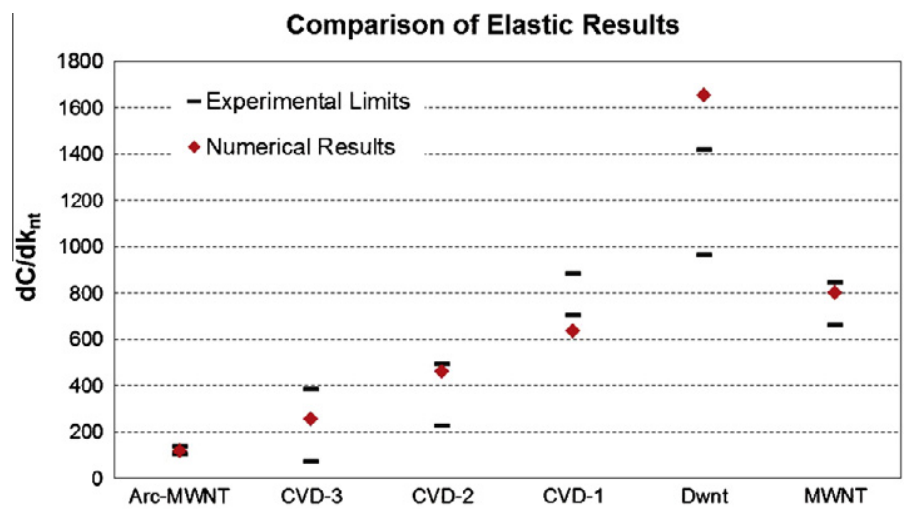

Fig. 5. Comparison of numerical and experimental results $[35,45]$.
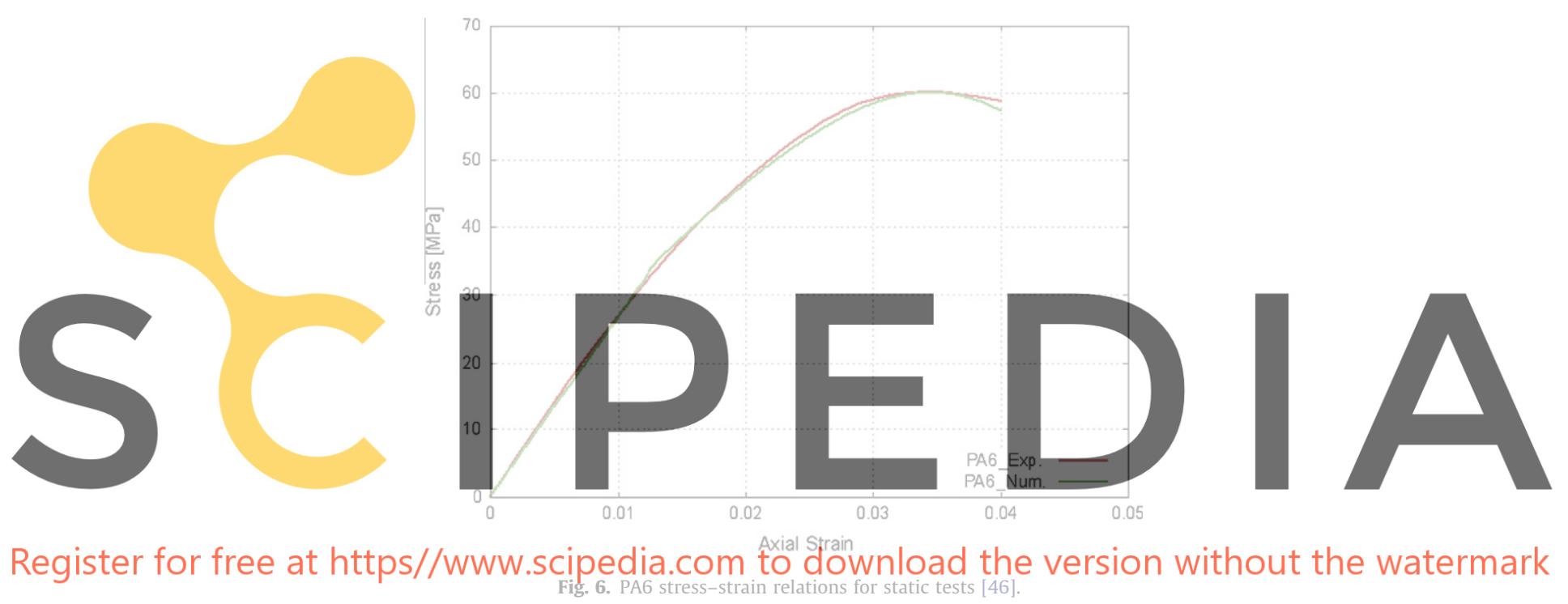

Table 2

Data of the composites.

\begin{tabular}{lllllll}
\hline Composite & $k_{n t}(\%)$ & $k_{i z}(\%)$ & $k_{m}(\%)$ & $l_{n t} / d_{n t}$ & $b / r_{n t}$ & $N^{\text {par }}$ \\
\hline PA6/A-MWCNT & 0.5 & 4.1 & 95.4 & 250 & 2.00 & 0.98 \\
PA6/U-MWCNT & 0.5 & 5.3 & 94.2 & 250 & 2.35 & 0.98 \\
\hline
\end{tabular}

the three composite components in each layer. This table also shows some geometry information of the MWCNTs and the interface zone, as well as the initial value of $N^{\text {par }}$.

In Fig. 7 are represented the numerical and experimental results obtained for the composite made with A-MWCNTs. The mechanical properties of each composite component are those defined previously.

This figure shows an initial reduction of the composite stiffness, result of matrix yielding. Afterwards damage begins in the interface zone and, consequently, the composite continues reducing its stiffness. Interface damage leads to a reduction of the parallel length (Eq. (42)). When the interface is completely damaged, the whole CNT-interface material has a serial performance. At this stage stresses in the interface are zero, and so must be the stresses in the carbon nanotube. Therefore, the final stiffness of the composite corresponds to a material with a volumetric participation of $95.4 \%$ of PA6 matrix, and the rest of the material correspond to voids.

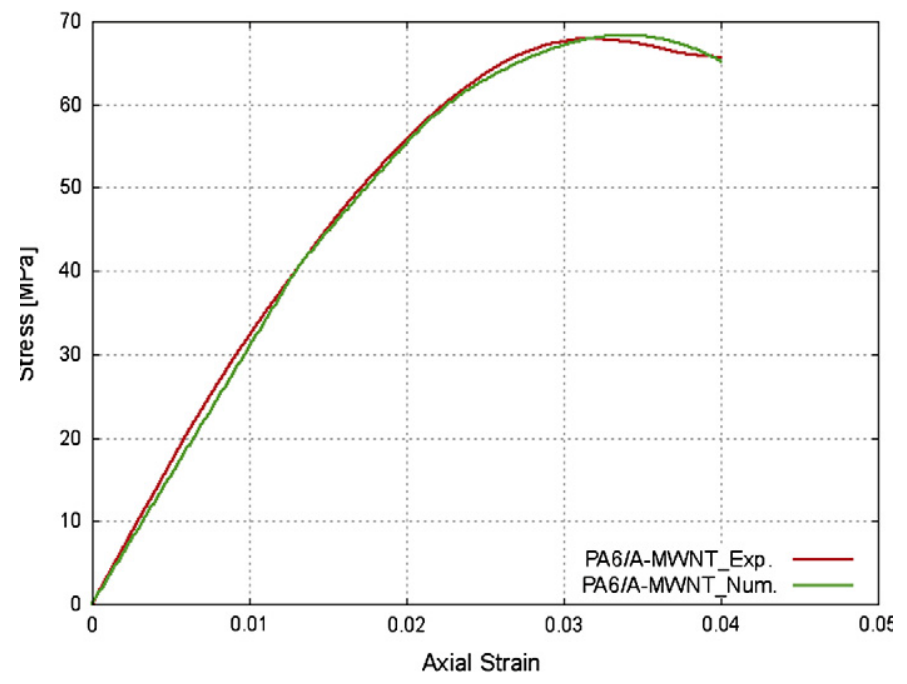

Fig. 7. PA6/A-MWCNT stress-strain relations for static tests [46].

Fig. 8 shows the results for the composite made with UMWCNTs. This composite is the same than the previous one (made with A-MWCNTs), with the only difference that in this case the bond between U-MWCNTs and interface zone is weaker. To take 


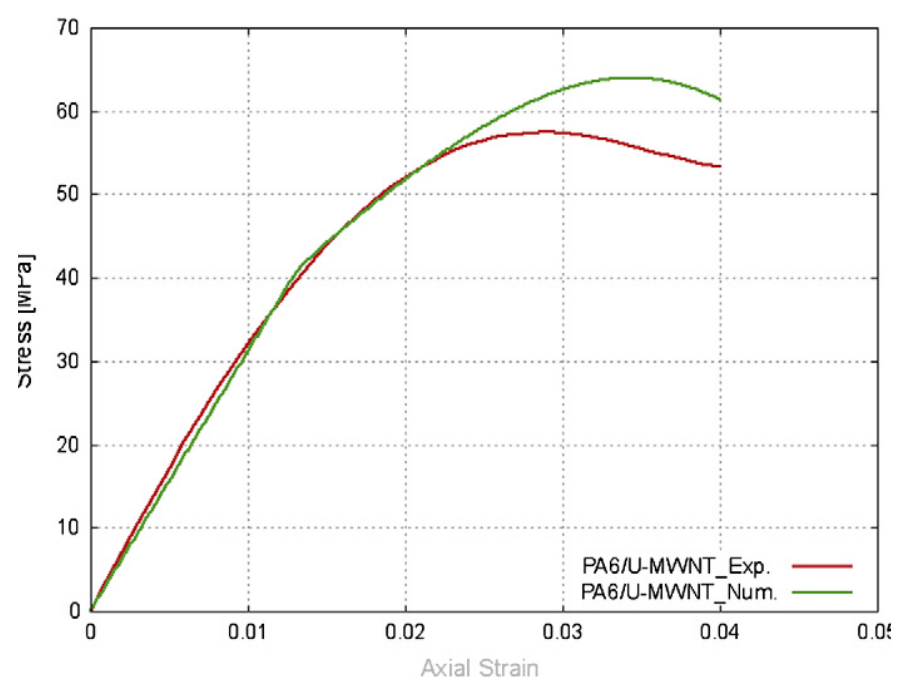

Fig. 8. PA6/U-MWCNT stress-strain relations for static tests [46].

into account this difference, the numerical model used for this composite is the same used for the previous one, varying the threshold at which damage starts in the interface. In current simulation this value is reduced to $70(\mathrm{MPa})$.

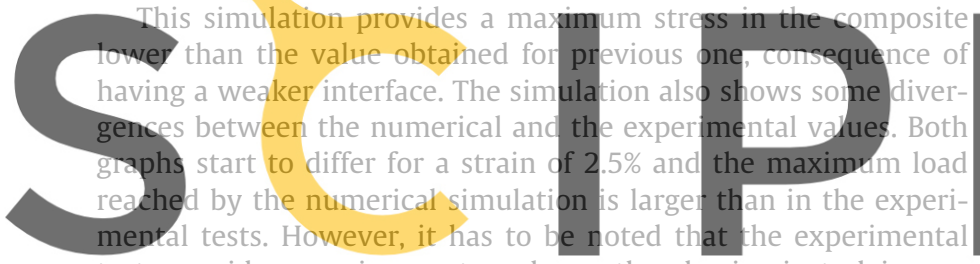
tests provide a maximum stress lower than having just plain matrix (Fig. 6). Therefore, the differences observed in Fig. 8 may be Registeififor free at https//www.scipedia.com to

\section{Conclusions}

The present paper has presented a new formulation, based on the mixing theory, capable of predicting the mechanical performance of composites reinforced with carbon nanotubes. The model presented relates the CNTs and the matrix in which they are embedded, using an interface material. This approach makes possible to consider non-linear phenomenons, such as CNT debounding, by using non-linear constitutive laws to characterize the interface material. The formulation is written in a way in which all materials can be defined with their own constitutive law, improving the versatility of the model.

It has been shown that the elastic properties estimated with the model are in good agreement with experimental values obtained from literature. Only the model of the composite made with the Dwnt reinforcement has given results in which the composite stiffness is overestimated. This is because the Dwnt has a very small diameter, which leads to a very high value of its equivalent Young's modulus.

The validation of the non-linear response provided by the new formulation has been performed using the experimental data of two different composites made with MWCNTs randomly distributed. The numerical curve obtained for the A-MWCNT is in good agreement with the experimental results. On the other hand, the numerical prediction obtained for the U-MWCNTs differs from the experimental results for strains larger than $2.5 \%$. However, it has to be said that the experimental results are lower than expected, as this composite is weaker than plain matrix.

\section{Acknowledgements}

This work has been supported by the European Community under Grant 246067, Multiscale Reinforcement of Semi-crystalline Thermoplastic Sheets and Honeycombs (M_RECT), NMP-20092.5-1; and by the Spanish Government, Secretaria de Estado de Investigacin, Desarrollo e Innovacin, through the project, Comportamiento no-lineal de materiales compuestos multilaminados, considerando la delaminacin (DELCOM), Ref. MAT2008-02232. All this support is gratefully acknowledged.

\section{References}

[1] Barbero EJ. Introduction to composite materials design. 2nd ed. Taylor \& Francis; 2011.

[2] Jayatilaka AS. Fracture of engineering brittle materials. London: Applied Sciences; 1979

[3] Halpin JC, Kardos JL. The halpin-tsai equations: a review. Polym Eng Sci $1976 ; 16(5): 344-52$.

4] Truesdell CA, Toupin R. The classical field theories. Handbuch der Physik, vol. 3. Berlin: Springer-Verlag; 1960. pp. 226-793 [chapter 1].

[5] Lijima S. Helical microtubules of graphitic carbon. Nature 1991;354:56-8.

[6] Coleman JN, Khan U, Blau WJ, Gunko YK. Small but strong: a review of the mechanical properties of carbon nanotube polymer composites. Carbon 2006;44(9):1624-52.

[7] Ruoff RS, Lorents DC. Mechanical and thermal properties of carbon nanotubes. Carbon 1995;33(7):925-30

[8] Salvetat J-P, Bonard J-M, Thomson NH, Kulik AJ, Forró L, Benoit W, et al. Mechanical properties of carbon nanotubes. Appl Phys A: Mater Sci Process 1999;69(3):255-60.

9] Cadek M, Murphy R, McCarthy B, Drury A, Lahr B, Barklie RC, et al. Optimisation

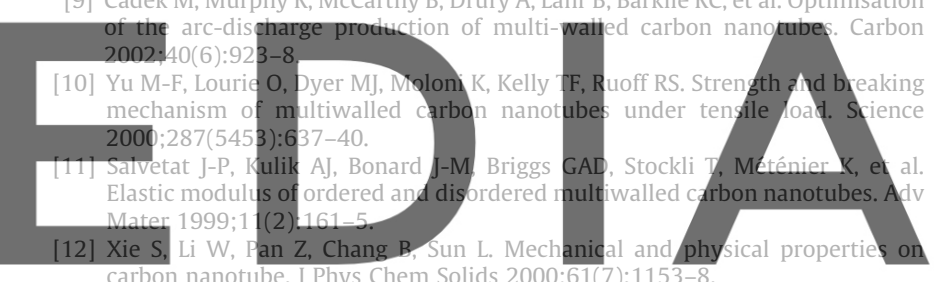

13] Cadek M, Coleman JN Ryan KP, Nicolosi V, Bister G, Fonseca A, et at.

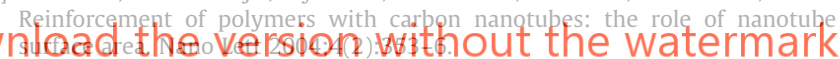

[14] McCarthy B, Coleman JN, Czerw R, Dalton AB, in het Panhuis M, Maiti A, et al. A microscopic and spectroscopic study of interactions between carbon nanotubes and a conjugated polymer. J Phys Chem B 2002;106(9): 2210-6.

[15] McCarthy B, Coleman JN, Curran SA, Dalton AB, Davey AP, Konya Z, et al. Observation of site selective binding in a polymer nanotube composite; 2000.

[16] Yang M, Koutsos V, Zaiser M. Interactions between polymers and carbon nanotubes: a molecular dynamics study. J Phys Chem B 2005;109(20):10009-14.

[17] Tsuda T, Ogasawara T, Deng F, Takeda N. Direct measurements of interfacial shear strength of multi-walled carbon nanotuve/peek composite using a nanopullout method. Compos Sci Technol 2011;7:1295-300.

[18] Cooper CA, Cohen SR, Barber AH, Wagner HD. Detachment of nanotubes from a polymer matrix. Appl Phys Lett 2002;81(20):3873-5.

[19] Barber AH, Cohen SR, Wagner HD. Measurement of carbon nanotube polymer interfacial strength. Appl Phys Lett 2003;82(23):4140-2.

[20] Ding W, Eitan A, Fisher FT, Chen X, Dikin DA, Andrews R, et al. Direct observation of polymer sheathing in carbon nanotube polycarbonate composites. Nano Lett 2003;3(11):1593-7.

[21] Sandler J, Werner P, Shaffer MSP, Demchuk V, Altstdt V, Windle AH. Carbonnanofibre-reinforced poly(ether ether ketone) composites. Compos Part A: Appl Sci Manuf 2002;33(8):1033-9.

[22] Sandler J, Broza G, Nolte M, Schulte K, Lam Y-M, Shaffer MSP, et al. Crystallization of carbon nanotube and nanofiber polypropylene composites. J Macromol Sci, Part B: Phys 2003;42(3):479.

[23] Wagner HD, Lourie O, Feldman Y, Tenne R. Stress-induced fragmentation of multiwall carbon nanotubes in a polymer matrix. Appl Phys Lett 1998;72(2):188-90.

[24] Frankland SJV, Caglar A, Brenner DW, Griebel M. Molecular simulation of the influence of chemical cross-links on the shear strength of carbon nanotubepolymer interfaces. J Phys Chem B 2002;106(12):3046-8.

[25] Garg A, Sinnott SB. Effect of chemical functionalization on the mechanical properties of carbon nanotubes. Chem Phys Lett 1998;295(4):273-8.

[26] Liao K, Li S. Interfacial characteristics of a carbon nanotube-polystyrene composite system. Appl Phys Lett 2001;79(25):4225-7.

[27] Lordi V, Yao N. Molecular mechanics of binding in carbon-nanotube-polymer composites. J Mater Res 2000;15(12):2770-9. 
[28] Barber AH, Cohen SR, Kenig S, Wagner HD. Interfacial fracture energy measurements for multi-walled carbon nanotubes pulled from a polymer matrix. Compos Sci Technol 2004;64(15):2283-9.

[29] Hwang GL, Shieh Y-T, Hwang KC. Efficient load transfer to polymer-grafted multiwalled carbon nanotubes in polymer composites. Adv Funct Mater 2004;14(5):487-91.

[30] Xia H, Wang Q Qiu G. Polymer-encapsulated carbon nanotubes prepared through ultrasonically initiated in situ emulsion polymerization. Chem Mater 2003;15(20):3879-86.

[31] Lou X, Detrembleur C, Sciannamea V, Pagnoulle C, Jérome R. Grafting of alkoxyamine end-capped (co)polymers onto multi-walled carbon nanotubes. Polymer 2004;45(18):6097-102.

[32] Bhattacharyya S, Sinturel C, Salvetat JP, Saboungi M-L. Protein-functionalized carbon nanotube polymer composites. Appl Phys Lett 2005;86(11):113104.

[33] Deng F, Ogasawara T, Takeda N. Tensile properties at different temperature and observation of micro deformation of carbon nanotubes poly(ether ether ketone) composites. Compos Sci Technol 2007;67(14):2959-64.

[34] Car E, Oller S, Oñate E. An anisotropic elastoplastic constitutive model for large strain analysis of fiber reinforced composite materials. Comput Meth Appl Mech Eng 2000;185(2-4):245-77.

[35] Coleman JN, Cadek M, Ryan KP, Fonseca A, Nagy JB, Blau WJ, et al. Reinforcement of polymers with carbon nanotubes. The role of an ordered polymer interfacial region. Experiment and modeling. Polymer 2006;47(26):8556-61.

[36] Cadek M, Coleman JN, Barron V, Hedicke K, Blau WJ. Morphological and mechanical properties of carbon nanotube reinforced semicrystalline and amorphous polymer composites. Appl Phys Lett 2002;81(27):5123-5.

[37] Malvern LE. Introduction to the mechanics of a continuous medium; 1969.
[38] Oller S, Oñate E, Miquel J, Botello S. A plastic damage constitutive model for composite materials. Int J Solids Struct 1996;33(17):2501-18.

[39] Lubliner J, Oliver J, Oller S, Oñate E. A plastic damage model for concrete. Int J Solids Struct 1989;25(3):299-326.

[40] Thostenson ET, Chou T. On the elastic properties of carbon nanotube-based composites: modelling and characterization. J Phys D: Appl Phys 2003;36: 573-82.

[41] Zhou X, Shin E, Wang KW, Bakis CE. Interfacial damping characteristics of carbon nanotube-based composites. Compos Sci Technol 2004;64:2425-37.

[42] PLCD Manual. Non-linear thermomachanic finite element code oriented to PhD student education. Code developed at CIMNE; 1991-to present.

[43] Martinez X, Oller S, Rastellini F, Barbat AH. A numerical procedure simulating rc structures reinforced with frp using the serial/parallel mixing theory. Comput Struct 2008;86:1604-18.

[44] Rastellini F, Oller S, Salomón O, Oñate E. Composite materials non-linear modelling for long fibre-reinforced laminates continuum basis, computational aspect and validations. Comput Struct 2008;86:879-96.

[45] Coleman JN, Cadek M, Blake R, Nicolosi V, Ryan KP, Belton C, et al. High performance nanotube-reinforced plastics: understanding the mechanism of strength increase. Adv Funct Mater 2004;14(8):791-8.

[46] Meng H, Sui GX, Fang PF, Yang R. Effects of acid- and diamine-modified mwnts on the mechanical properties and crystallization behavior of polymide 6 . Polymer 2008;49:610-20.

[47] Li C, Chou T. Elastic moduli of multi-walled carbon nanotubes and the affect of van der waals forces. Compos Sci Technol 2003;63:1517-24.

[48] Maurin R, Davies P, Baral N, Baley C. Transverse properties of carbon fibres by nano-indentation and micro-mechanics. Appl Comp Mater 2008;15(2): $61-73$. 